\title{
Levosimendan accelerates recovery in patients with takotsubo cardiomyopathy
}

\author{
Mehmet Yaman ${ }^{1,2,3}$, Ugur Arslan'², Ahmet Kaya ${ }^{1,4}$, Aytac Akyol ${ }^{3,5}$, \\ Fatih Ozturk ${ }^{3}$, Yunus Emre Okudan ${ }^{6}$, Adil Bayramoglu ${ }^{1}$, Osman Bektas ${ }^{1}$ \\ ${ }^{1}$ Cardiology Department, Ordu University Education and Research Hospital, Ordu, Turkey \\ ${ }^{2}$ Cardiology Department, Samsun Education and Research Hospital, Samsun, Turkey \\ ${ }^{3}$ Cardiology Department, Van Yuzuncu Y1l University Medicine Faculty, Van, Turkey \\ ${ }^{4}$ Cardiology Department Erzurum Region Education and Research Hospital, Erzurum, Turkey \\ ${ }^{5}$ Cardiology Department, Van Region Education and Research Hospital, Van, Turkey \\ ${ }^{6}$ Cardiology Department Suleyman Demirel University Medicine Faculty, Isparta, Turkey
}

\begin{abstract}
Background: The aim of this study was to determine the efficacy and safety of levosimendan in takotsubo cardiomyopathy (TC).

Methods: The study was conducted in a retrospective design and 42 consecutive patients were enrolled in 6 cardiovascular centers in Turkey. The records of TC patients having left ventricular ejection fraction (LVEF) $\leq 35 \%$ were examined at admission, discharge and recovery period including their clinical and echocardiographic data.

Results: Of these 42 TC patients, 17 were treated with loading dose and i.v. infusion of levosimendan (group 1) and 25 were treated without levosimendan (group 2). Echocardiographic findings at admission and at discharge were similar and no serious complications were observed in either group. However recovery period including the interval of $50 \%$ increase in $L V E F$, time to achieve the baseline troponin values and hospitalization were significantly lower in patients taking levosimendan.

Conclusions: This is the first study using loading dose and subsequent continuous intravenous administration of levosimendan demonstrating accelerated recovery in patients with TC. (Cardiol J 2016; 23, 6: 610-615)
\end{abstract}

Key words: takotsubo cardiomyopathy, levosimendan, recovery, heart failure, stress cardiomyopathy

\section{Introduction}

Takotsubo cardiomyopathy (TC), also known as stress cardiomyopathy, left ventricular (LV) apical ballooning syndrome or broken heart syndrome, is a unique cardiac situation characterized by transient LV systolic dysfunction often mimicking an acute myocardial infarction. In most cases the un- derlying factor can be elaborated as acute emotional and/or physical stress and it is characterized by three distinctive features: (a) the presence of acute LV wall dysfunction, (b) the absence of significant obstructive coronary artery disease and (c) the rapid improvement of LV systolic function within a few days or weeks $[1,2]$. The clinical presentation of $\mathrm{TC}$ causes a significant risk for adverse events

Address for correspondence: Mehmet Yaman, MD, Ordu University Education and Research Hospital Cardiology Department, 52100 Ordu, Turkey, tel: +905334774146, fax: +904522454144, e-mail: dr.yaman@windowslive.com 
and thus represents an acute heart failure [3]. Although the acute course of TC is not malignant in most cases, $20 \%$ face some complications such as ventricular tachycardia, ventricular thrombus and ventricular rupture similar to, or higher than those of patients with acute coronary syndrome during their hospital stay.

The underlying mechanism of TC still remains unclear but many hypotheses including multivessel coronary artery spasm, coronary microvascular dysfunction or catecholamine toxicity, have been introduced. In the clinical setting, $\mathrm{TC}$ is an important disease that must be promptly differentiated from acute myocardial infarction to enable its appropriate management [4].

Levosimendan is a powerful inhibitor of phosphodiesterases but its positive inotropic effect is achieved without a significant increase of intracellular cAMP and $\mathrm{Ca}^{2+}$. This calcium-sensitizer drug reinforces the $\mathrm{Ca}^{2+}$-binding with troponin-C during systole and improves ventricular relaxation during diastole as the so-called lusitropic effect; moreover, this levosimendan shows important anti-stunning and vasodilator effects by opening the ATP-sensitive $\mathrm{K}^{+}$-channels [5]. Previously levosimendan had been proposed as the choice of inotropic drug by Santoro et al. [6] and to our knowledge, levosimendan was the pivotal therapeutic agent in treatment of TC. Herein, we retrospectively compared the acute course of TC patients treated versus untreated with the loading dose and infusion of levosimendan.

\section{Methods}

A total of 42 subjects were enrolled in a restrospective manner between January $1^{\text {st }} 2010$ to July $31^{\text {st }} 2016$ at 6 centres in Turkey. The records of $42 \mathrm{TC}$ patients having $\mathrm{LV}$ ejection fraction (LVEF) $\leq 35 \%$ were examined. Mayo diagnostic criteria [7] was utilized where all the four items were mandatory for accurate diagnosis:

- Transient LV dysfuntion (hypokinesis, akinesis, or dyskinesis): The wall motion abnormalities are typically regional and extend beyond a single epicardial coronary distribution; rare exceptions are the focal (within one coronary distribution) and the global type;

- Absence of obstructive coronary disease or angiographic evidence of acute plaque rupture: If coronary disease is found, the diagnosis of stress cardiomyopathy can still be made if the wall motion abnormalities are not in the distribution of the coronary disease. This exception is made since some patients with stress cardiomyopathy have concurrent coronary disease; - New electrocardiographic abnormalities (either ST-segment elevation and/or T wave inversion) or modest elevation in cardiac troponin;

- Absence of pheochromocytoma or myocarditis. Of the 42 patients, 17 patients (female: 12 ) were treated with intravenous (i.v.) continuous infusion of levosimendan within the first $24 \mathrm{~h}$ after admission in 2 centres as a routine therapy; infusion rate was $0.1 \mu \mathrm{g} / \mathrm{kg} / \mathrm{min}$ with $10 \mu \mathrm{g} / \mathrm{kg}$ loading dose for $24 \mathrm{~h}$. The loading dose was used as a standard levosimendan therapy for all patients without hypotension (systolic/diastolic blood pressure $<90 / 60 \mathrm{~mm} \mathrm{Hg}$ ). Twenty five patients (female, $\mathrm{n}=18$ ) were treated without i.v. continuous infusion of levosimendan in the other 4 centres. All patients were administered a standard of care including angiotensin converting enzyme inhibitor/angiotensin receptor blockers and beta-blocker unless contraindicated. Medical records, including medical history, physical examination, laboratory tests, coronary angiography, 12-lead electrocardiogram (ECG) also echocardiographic findings for cases in which they were available were carefully reviewed. The following data were obtained by analyzing age, gender, physical - emotional stress factors, days of hospitalization and coronary risk factors including smoking, hypertension (as defined by the Joint National Committee VIII) [8], diabetes mellitus (as defined by the World Health Organization study group) [9], dyslipidemia was considered present if the total cholesterol concentration on admission was higher than $220 \mathrm{mg} / \mathrm{dL}$ or the low-density lipoprotein cholesterol concentration on admission was higher than $140 \mathrm{mg} / \mathrm{dL}$ and a family history of premature coronary artery disease defined as myocardial infarction or sudden death in a first relative, male $<55$ years and female $<65$ years.

All patients underwent continuous ECG and hemodynamic monitoring for $48 \mathrm{~h}$. Troponin I levels were measured for all patients using a chemiluminescent immunoenzymatic assay (Access AccuTnI, Beckman Coulter, Fullerton, CA, USA). The detection limit of this assay is $0.01 \mathrm{ng} / \mathrm{mL}$ and the $99^{\text {th }}$ percentile in an apparently healthy reference population is reported to be $0.04 \mathrm{ng} / \mathrm{mL}$. Patients underwent coronary angiography procedure which showed the absence of coronary disease. Transthoracic echocardiography examination was performed in all patients at admission, during the hospital stay and at discharge. LVEF was calculated using the biplane method (modified Simpson's rule) 
Table 1. Baseline characteristics.

\begin{tabular}{lccc}
\hline Variable & Group 1 (n = 17) & Group 2 (n = 25) & $\mathbf{P}$ \\
\hline Age [years] & $59.2 \pm 10.9$ & $63.3 \pm 6.5$ & 0.140 \\
Sex (female) & $12(70.6 \%)$ & $18(72 \%)$ & 0.065 \\
Body mass index $\left[\mathrm{kg} / \mathrm{m}^{2}\right]$ & $26.7 \pm 4.1$ & $28.6 \pm 4.5$ & 0.186 \\
Hypertension & $5(29.4 \%)$ & $9(36 \%)$ & 0.666 \\
Dyslipidemia & $1(5.9 \%)$ & $1(4 \%)$ & 0.143 \\
Diabetes mellitus & $5(29.4 \%)$ & $5(20 \%)$ & 0.494 \\
Smoking & $7(41.2 \%)$ & $13(52 \%)$ & 0.503 \\
Emotional stress & $13(76.5 \%)$ & $15(60 \%)$ & 0.082 \\
Physical stress & $4(23.5 \%)$ & $10(40 \%)$ & 0.065 \\
Systolic arterial pressure $[\mathrm{mm} \mathrm{Hg]}$ & $115 \pm 28$ & $118 \pm 25$ & 0.176 \\
Diastolic arterial pressure [mm Hg] & $73 \pm 11$ & $74 \pm 10$ & 0.172 \\
Heart rate [bpm] & $88 \pm 25$ & $86 \pm 27$ & 0.184 \\
Troponin levels [ng/dL] & $6.9 \pm 3.1$ & $7.3 \pm 3.0$ & 0.628 \\
Medications: & & & 0.147 \\
ACEl/ARB & $17(100 \%)$ & $24(96.0 \%)$ & 0.068 \\
Beta-blocker & $15(88.2 \%)$ & $21(84.0 \%)$ & $12(48.0 \%)$ \\
Spironolactone & $8(47.1 \%)$ & $3(12.0 \%)$ & 0.854 \\
Diuretic (furosemide) & $2(11.8 \%)$ & $0(0 \%)$ & $<012$ \\
(+) inotropes including dopamine and dobutamine & $1(5.9 \%)$ & 0.001 \\
\hline
\end{tabular}

ACEI — angiotensin converting enzyme inhibitor; ARB — angiotensin receptor blockers

from the apical 4-chamber and 2-chamber views. LVEF recovery to $>50 \%$ was the only criterion for discharge from hospital. The study protocol was in agreement with the guidelines of the ethics committee of aforementioned institutions.

\section{Statistical analysis}

All data are expressed as mean \pm standard deviation. Distributions of continuous variables in the two groups were compared with either the unpaired t test or the Mann-Whitney U-test according to whether data followed the normal distribution. Comparison between the two groups was performed by using the $\chi^{2}$ test for categorical variables. ANOVA test was used where $>$ two groups were present to compare. Missing values were replaced by multiple regression imputation. All tests were performed with IBM SPSS for Windows, version 21.0. (Chicago, USA). All results were considered statistically significant at the level of $p<0.05$.

\section{Results}

Retrospectively 42 consecutive patients were enrolled and diagnosed with $\mathrm{TC}$ and of these, 17 were treated with levosimendan (group 1) and
25 were treated without levosimendan (group 2). Baseline characteristics listed in Table 1 were similar in both groups. Triggers of TC such as physical and emotional stress were also similar in both groups $(\mathrm{p}=0.453)$.

Only 1 patient was admitted with cardiogenic shock and she was treated with levosimendan plus dopamine. Fortunately she has recovered quite well. No serious complications including arrhythmia were observed in either groups.

Echocardiographic findings at admission and discharge were similar in both groups (Table 2). In group 1 , the mean LVEF at admission was $32.3 \pm 3.1 \%$ and $32.0 \pm 3.8 \%$ in group $2(\mathrm{p}=0.754)$. All patients showed improvement in LVEF during discharge where mean LVEF at discharge was $57.3 \pm$ $\pm 5.0 \%$ in group 1 and $55.2 \pm 4.4 \%$ in group 2 $(\mathrm{p}=0.152)$.

However, the recovery period significantly differs between the two groups (Table 3 ). The time to LVEF rise above $50 \%$ was significantly lower in group 1 compared to group $2(8.3 \pm 1.9$ days vs. $10.8 \pm 3.1$ days, $\mathrm{p}=0.001$ ). Hospitalization time also showed statistical significance (lower in group 1) compared to group $2(9.4 \pm 1.7$ days vs. $14.3 \pm 1.5$ days, $\mathrm{p}<0.001)$. The recovery time to baseline 
Table 2. Echocardiographic findings at admission and at discharge.

\begin{tabular}{|c|c|c|c|c|c|}
\hline & \multicolumn{2}{|c|}{ Group $1(n=17)$} & \multicolumn{2}{|c|}{ Group $2(n=25)$} & \multirow[t]{2}{*}{$\mathbf{P}$} \\
\hline & Admission & Discharge & Admission & Discharge & \\
\hline AoD $[\mathrm{mm}]$ & $27.0 \pm 2.3$ & $26.9 \pm 2.8$ & $29.2 \pm 1.4$ & $25.8 \pm 2.8$ & 0.216 \\
\hline LAD [mm] & $38 \pm 2.7$ & $35.9 \pm 1.8$ & $32 \pm 2.7$ & $37.1 \pm 4.6$ & 0.306 \\
\hline LVEDD [mm] & $51.6 \pm 2.1$ & $50.1 \pm 2.6$ & $51.1 \pm 6.1$ & $49.3 \pm 6.1$ & 0.635 \\
\hline LVESD [mm] & $36.0 \pm 3.2$ & $31.0 \pm 1.8$ & $34.1 \pm 9.1$ & $30.4 \pm 8.3$ & 0.430 \\
\hline IVSD [mm] & $10.3 \pm 1.1$ & $10.9 \pm 3.8$ & $10.2 \pm 1.2$ & $10.8 \pm 3.6$ & 0.143 \\
\hline PWD [mm] & $10.5 \pm 1.8$ & $10.7 \pm 3.4$ & $10.8 \pm 2.3$ & $10.9 \pm 2.9$ & 0.096 \\
\hline LVEF [\%] & $32.3 \pm 3.1$ & $57.3 \pm 5.0$ & $32.0 \pm 3.8$ & $55.2 \pm 4.4$ & 0.152 \\
\hline
\end{tabular}

AoD - aortic root diameter; LAD — left atrial diameter; LVEDD — left ventricular end-diastolic diameter; LVESD — left ventricular end-systolic diameter; IVSD — interventricular septum diastolic dimension; PWD — posterior wall dimension; LVEF — left ventricular ejection fraction

Table 3. Recovery of patients in both groups.

\begin{tabular}{lccc}
\hline Variable & $\begin{array}{c}\text { Group 1 } \\
(\mathbf{n}=\mathbf{1 7})\end{array}$ & $\begin{array}{c}\text { Group 2 } \\
(\mathbf{n = 2 5})\end{array}$ & $\mathbf{P}$ \\
\hline Hospital stay [days] & $9.4 \pm 1.7$ & $14.3 \pm 1.5$ & $<\mathbf{0 . 0 0 1}$ \\
Time to recover to the baseline troponin values [days] & $5.1 \pm 1.6$ & $8.2 \pm 2.4$ & $\mathbf{0 . 0 0 1}$ \\
Time to rise in left ventricular ejection fraction above 50\% [days] & $8.3 \pm 1.9$ & $10.8 \pm 3.1$ & $\mathbf{0 . 0 0 1}$ \\
\hline
\end{tabular}

troponin values were also significantly lower in group 1 compared to group $2(5.1 \pm 1.6$ days vs. $8.2 \pm 2.4$ days, $\mathrm{p}=0.001$ ).

\section{Discussion}

This study evaluated the efficacy of the treatment strategy with levosimendan compared to standard therapy in TC patients with severe LV dysfunction independent of baseline blood pressure. Actually, this is the first study investigating the efficacy of loading dose followed by the i.v. continuous infusion of levosimendan in TC setting. We have found that all patients were safely treated with i.v. infusion of levosimendan and also it has reduced hospital stay and recovery to baseline clinical parameters.

In the literature, there were only a few levosimendan (without loading dose) treated TC cases that were complicated with cardiogenic shock [10-13]. However in this present study, none of the patients in both groups were complicated by cardiogenic shock and all were treated with loading dose of levosimendan. In a comprehensive case series recently published by Santoro et al. [6], it was evaluated that the use of levosimendan without loading dose in patients with TC might be safe and feasible with low rates of adverse events. Herein, we used loading dose and found similar results with this study. Santoro et al. [6] have also demonstrated that mean hospital stay of patients treated with levosimendan was $10 \pm$ \pm 4 days and was quite similar to results found in this study $(9.4 \pm 1.7$ days). Additional findings disclosed that time to recovery to the baseline troponin values and time to LVEF rise above $50 \%$ were significantly lower in patients treated with levosimendan.

Levosimendan infusion was well tolerated in group 1 patients. No malignant cardiac arrhythmias were observed and none of the patients developed LV outflow tract obstruction. LVEF improved in all patients independent of treatment with levosimendan, however LVEF improvement was faster in the patients treated with levosimendan.

Levosimendan is a new molecule with both inotropic and vasodilator effect. The main mechanism of action described for this drug is elaborated as the increase in the troponin $\mathrm{C}$ affinity for $\mathrm{Ca}^{2+}$ and the stabilization of troponin $\mathrm{C}$ conformation. The administration of this calcium sensitizer presents other effects such as peripheral vasodilatation, antiischaemic benefits and cardioprotection [14]. The main mechanism of increasing myocardial contractilityis based on the increase of sensitivity of cardiac troponin $\mathrm{C}$ towards intra-cytoplasmic calcium [15-18]. Experimental studies have demonstrated that levosimendan has a preconditioning-like effect 
on myocardial function [19] as it protects the heart against ischaemia and reperfusion damage by the opening of cardiac mitochondrial ATP-sensitive potassium [mito.K (ATP)] channels, one of the main mediators of cell protection pathways [20]. This mechanism of positive inotrophic myocardial contraction strengthening occurs without increasing oxygen demand, intracellular cAMP or intracellular calcium concentration at clinically relevant doses [21-23]. Levosimendan activates the opening of ATP-sensitive $\mathrm{K}^{+}$channels of smooth muscle cells allowing their hyperpolarization by leveraging vasodilation $[24,25]$. These vasodilatory properties lead to a dramatic increase in cardiac output with a concomitant reduction in cardiac filling pressures in the failing heart to enable it to generate more efficient systolic and diastolic functions [26, 27]. All these mechanisms mentioned above may contribute to more rapid improvement of ventricular functions and clinical recovery in patients who were treated with levosimendan in this study.

There are case reports of successful levosimendan use in cardiomyopathy due to acute Chagas' disease, peri-partum cardiomyopathy, anthracycline-induced cardiotoxicity and TC $[6,10-13,28-30]$. In the present study, it was demonstrated that levosimendan can be used safely and efficiently in $\mathrm{TC}$ as a non-catecholaminergic inotrope agent for faster symptomatic and clinical improvement for the first time.

\section{Limitations of the study}

Our results indicate that levosimendan may have positive effects in TC with a loading dose safely and efficiently. The present study, however, has some limitations due to the small number of patients and its retrospective nature. Further prospective randomized studies enrolling more patients are needed to validate these results.

\section{Conclusions}

This is the first study using loading dose and subsequent continuous intravenous administration of levosimendan demonstrating accelerated recovery in patients with $\mathrm{TC}$.

\section{Conflict of interest: None declared}

\section{References}

1. Kurisu S, Sato H, Kawagoe T et al. Tako-tsubo-like left ventricular dysfunction with ST segment elevation: A novel cardiac syndrome mimicking acute myocardial infarction. Am Heart J, 2002; 143: 448-455.

2. Prasad A, Lerman A, Rihal CS. Apical ballooning syndrome (Tako-tsubo or stress cardiomyopathy): A mimic of acute myocardial infarction. Am Heart J, 2008; 155: 408-417.

3. Templin C, Ghadri JR, Diekmann J et al. Clinical features and outcomes of takotsubo (stress) cardiomyopathy. N Engl J Med, 2015; 373: 929-938.

4. Kurisu S, Kihara Y. Clinical management of takotsubo cardiomyopathy. Circ J, 2014; 78: 1559-1566.

5. Antonini M, Stazi GV, Cirasa MT, Garotto G, Frustaci A. Efficacy of levosimendan in Takotsubo-related cardiogenic shock. Acta Anaesthesiol Scand, 2010; 54: 119-120.

6. Santoro F, Ieva R, Ferraretti A et al. Safety and feasibility of levosimendan administration in takotsubo cardiomyopathy: A case series. Cardiovasc Ther, 2013; 31: 133-137.

7. Bybee KA, Kara T, Prasad A et al. Systematic review: transient left ventricular apical ballooning: a syndrome that mimics ST segment elevation myocardial infarction. Ann Intern Med, 2004; 141: 858-865.

8. James PA, Oparil S, Carter BL et al. 2014 Evidence-based guideline for the management of high blood pressure in adults: Report from the panel members appointed to the Eighth Joint National Committee (JNC 8). JAMA, 2014; 311: 507-520.

9. Alberti KG, Zimmet PZ. Definition, diagnosis and classification of diabetes mellitus and its complications. Part 1: Diagnosis and classification of diabetes mellitus provisional report of a WHO consultation. Diabet Med, 1998; 15: 539-553.

10. Padayachee L. Levosimendan: The inotrope of choice in cardiogenic shock secondary totakotsubo cardiomyopathy? Heart Lung Circ, 2007;16 (suppl. 3): S65-S70.

11. De Santis V, Vitale D, Tritapepe L, Greco C, Pietropaoli P. Use of levosimendan forcardiogenic shock in a patient with the apical ballooning syndrome. Ann Intern Med, 2008; 149: 365-367.

12. Antonini M, Stazi GV, Cirasa MT, Garotto G, Frustaci A. Efficacy of levosimendan in Takotsubo-related cardiogenic shock. Acta Anaesthesiol Scand, 2010; 54: 119-120.

13. Karvouniaris M, Papanikolaou J, Makris D, Zakynthinos E. Sepsis-associated takotsubo cardiomyopathy can be reversed with levosimendan. Am J Emerg Med, 2012; 30: 832.e5-e7.

14. Pathak A, Lebrin M, Vaccaro A, Senard JM, Despas F. Pharmacology of levosimendan: inotropic, vasodilatory and cardioprotective effects. J Clin Pharm Ther, 2013; 38: 341-349.

15. Haikala H, Linden IB. Mechanisms of action of calcium-sensitizing drugs. J Cardiovasc Pharmacol, 1995; 26 (suppl. 1): 10-19.

16. Haikala H, Kaivola J, Nissinen E, Wall P, Levijoki J, Linden IB. Cardiac troponin $\mathrm{C}$ asa target protein for a novel calcium-sensi-tizing drug, levosimendan. J Mol Cell Cardiol, 1995; 27: 1859-1866.

17. Sorsa T, Heikkinen S, Abbott MB et al. Binding of levosimendan, a calcium sensitizer,to cardiac troponin C. J Biol Chem, 2001; 276: 9337-9343.

18. Hasenfuss G, Pieske B, Castell M, Kretschmann B, Maier LS, Just H. Influence of thenovel inotropic agent levosimendan on isometric tension and calcium cycling in failing human myocardium. Circulation, 1998; 98: 2141-2147.

19. Kersten JR, Montgomery MW, Pagel PS, Warltier DC. Levosimendan, a new positiveinotropic drug, decreases myocardial infarct size via activation of K(ATP) channels. Anesth Analg, 2000; 90: 5-11. 
20. Eisen A, Fisman EZ, Rubenfire M et al. Ischemic preconditioning: nearly two decadesof research. A comprehensive review. Atherosclerosis, 2004; 172: 201-210.

21. Parissis JT, Rafouli-Stergiou P, Paraskevaidis I, Mebazaa A. Levosimendan: from basicscience to clinical practice. Heart Fail Rev, 2009; 14: 265-275.

22. Haikala H, Pollesello P. Calcium sensitivity enhancers. Drugs, 2000; 3: 1199-1205.

23. Kass DA, Solaro RJ. Mechanisms and use of calcium-sensitizing agents in the failingheart. Circulation, 2006; 113: 305-15.

24. Yokoshiki H, Katsube Y, Sunagawa M, Sperelakis N. Levosimendan, a novel $\mathrm{Ca}^{2+}$ sensitizer, activates the glibenclamide-sensitive $\mathrm{K}+$ channel in rat arterial myocytes. Eur J Pharmacol, 1997; 333: 249-259.

25. Kaheinen P, Pollesello P, Levijoki J, Haikala H. Levosimendan increases diastolic coronary flow in isolated guinea-pig heart by opening ATP-sensitive potassium channels. J Cardiovasc Pharmacol, 2001; 37: 367-374.
26. Jorgensen K, Bech-Hanssen O, Houltz E, Ricksten SE. Effects of levosimendan on leftventricular relaxation and early filling at maintained preload and afterload conditionsafter aortic valve replacement for aortic stenosis. Circulation, 2008; 117: 1075$-1081$.

27. Parissis JT, Paraskevaidis I, Bistola V et al. Effects of levosimendan on right ventricularfunction in patients with advanced heart failure. Am J Cardiol, 2006; 98: 1489-1492.

28. Ronsoni RM, Feijo RV Jr, Melo LH et al. The use of Levosimendan for myocardiopathy due to acute Chagas' disease. Int J Cardiol, 2009; 136: 233-235.

29. Benezet-Mazuecos J, de la Hera J. Peripartum cardiomyopathy: A new successful setting for levosimendan. Int J Cardiol, 2008; 123: $346-347$.

30. Garcia JAP, Simvoulidis LFN, Salluh JIF et al. Levosimendan in acute decompensation of anthracycline-induced cardiotoxicity. Int J Cardiol, 2007; 118: 406-407. 